\title{
Metabolic syndrome in the era of COVID-19 outbreak: impact of lockdown on cardiometabolic health
}

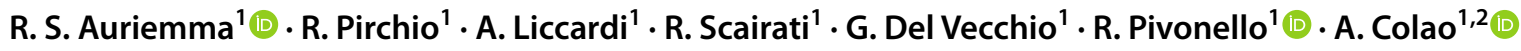

Received: 8 March 2021 / Accepted: 26 March 2021 / Published online: 26 May 2021

(c) The Author(s) 2021

\begin{abstract}
Purpose COVID-19 pandemics and cardiometabolic health are mutually interconnected. Chronic metabolic diseases are known risk factors for increased mortality after SARS-CoV-2 infection. In turn, COVID pandemics imposed sudden changes in lifestyle and social isolation with consequent potential cardiometabolic sequelae. The present study aimed at investigating the impact of changes in lifestyle and social life on metabolic profile in hyperprolactinemic or osteoporotic patients without pre-existing cardiometabolic diseases at the time of COVID-19.

Methods The primary study outcome measurement was the prevalence of obesity, arterial hypertension, impaired glucose tolerance (IGT) or diabetes mellitus (DM), dyslipidemia and metabolic syndrome after COVID-19 outbreak. Seventy-four patients ( 21 men and 53 women, aged $51.8 \pm 17.8$ years) were admitted to the outpatient clinic of the Neuroendocrine Disease Unit at University "Federico II" of Naples, Italy, as per their routine clinical practice because of tumoral and non-tumoral hyperprolactinemia in 52 patients (70.3\%), and osteoporosis/osteopenia in 22 (29.7\%). Among female patients, 25 (47.2\%) were at menopausal age.

Results At the end of lockdown, prevalence of obesity (from $37.8 \%$ to $51.3 \%, p<0.0001$ ), dyslipidemia (from 28.4 to $48.6 \%$, $p=0.003$ ) and metabolic syndrome (from 14.9 to $27 \%, p<0.0001$ ) significantly increased compared to pre-COVID evaluation. No significant change was found in the prevalence of arterial hypertension and IGT/DM.

Conclusion SARS-CoV-2 outbreak has led to a rapid increase in the prevalence of metabolic syndrome, potentially contributing to the increased COVID-19 related mortality.
\end{abstract}

Keywords COVID-19 pandemics $\cdot$ Metabolic syndrome $\cdot$ Cardiometabolic risk $\cdot$ Restrictions $\cdot$ Changes in lifestyle $\cdot$ Social isolation

\section{Rapid communication}

Between March, the 9th 2020 and May, the 4th 2020 special restrictions with a complete lockdown have been imposed by the Italian Government to limit the outbreak of COVID19 pandemics in Italy. As a result, Italian people have been required to stay home for approximately 2 months; any physical exercise in public sites, such as gyms and parks, has been forbidden and most professional activities have

A. Colao

colao@unina.it

1 Dipartimento di Medicina Clinica e Chirurgia, Sezione di Endocrinologia, Università "Federico II" di Napoli, Naples, Italy

2 UNESCO Chair for Health Education and Sustainable Development, Federico II University, Naples, Italy been turned into a smart working modality, with the exception of employers for essential services, including hospitals and other healthcare, utilities such as electricity and water supply, law enforcement and firefighting, and mostly food services. Such restrictions have been importantly effective in reducing viral spread, with infection and mortality rates progressively decreasing up to reach a safer condition, to stop the lockdown and to restart most routine working activities and social life. On the other side, these restrictive measures negatively influenced the everyday life, not only for elderlies considered to be at high risk of COVID-19 infection mainly if affected with chronic cardiometabolic diseases, but also for young healthy people.

However, during the approximately 60 days of lockdown Italians have changed not only social and public behavior, but mainly their lifestyle. At one side, in the vast majority of cases, routine physical exercise has been strongly limited to 
a few-minute domestic in-house fitness or totally abandoned, and most people have adopted a sedentary behavior. On the other side, time availability and rediscovery of familial conviviality exerted a negative impact also on dietary behavior, as demonstrated by the wide use of home-made food mainly prepared by fat sources (fresh meat, eggs, butter, long-life milk), or by whole white flour and sugar, such as pizza, cakes, pasta and bread [1]. In turn, the use of fresh healthy food, mainly including cereals, fish, fruits and vegetables, has been reduced despite its permanent availability at food stores [1], thus leading to a limited adherence to the Mediterranean diet, known to exert strong cardioprotective effects [2]. Note to worth, consequences of such changes in lifestyle and nutritional habits are even more alarming in patients with pre-existing obesity, diabetes mellitus and metabolic syndrome (MetS), which in turn have been demonstrated to be predisposed to higher risk of mortality following SARSCoV-2 infection [3]. Social isolation and loneliness, known to be associated with increased mortality risk mainly among elderlies, has resulted in notable changes in patterns of daily living, mainly timing of meals and sleep, and altered circadian biology, which in turn might severely impact cardiometabolic health [4]. This evidence has raised the questions of whether these changes might impair metabolic profile also in healthy people not affected by cardiometabolic diseases at the time of COVID-19 outbreak, and whether metabolic impairment might be an aftermath, and not only a risk factor, of COVID-19 disease.

By the end of lockdown up to July, the 30th 2020, 74 patients, including 21 men (28\%) and 53 women ( $72 \%)$, aged $51.8 \pm 17.8$ years, were consecutively admitted to the outpatient clinic of the Neuroendocrine Disease Unit at University "Federico II" of Naples as per their routine clinical practice because of tumoral and non-tumoral hyperprolactinemia in 52 patients (70.3\%), and osteoporosis/osteopenia in 22 (29.7\%). Among female patients, 25 (47.2\%) women were at menopausal age. Based on power calculation and sample size analysis, a total of 66 patients were required for a statistical power of $80 \%$ at $5 \%$ significance set. Based on age quartiles, patients were stratified as group 1 (16-38.25 years, 19 patients), group 2 (38.36-50 years, 19 patients), group 3 (50.1-67.75 years, 19 patients), group 4 (67.76-90 years, 17 patients). As shown in Fig. 1, at last follow-up before COVID-19 pandemics (within 3-6 months), 28 (37.8\%) were obese, $30(40.5 \%)$ had arterial hypertension, 22 (29.7\%) impaired glucose tolerance (IGT) or overt diabetes mellitus (DM), and 21 (28.4\%) dyslipidemia. According to the International Diabetes Federation consensus criteria [5], overall MetS was found in 11 patients (14.9\%, Fig. 1).

Throughout the lockdown period, all patients maintained treatment schedules previously established on the basis of their pre-existing endocrine disease and none suddenly withdrew medical therapies for concomitant cardiometabolic

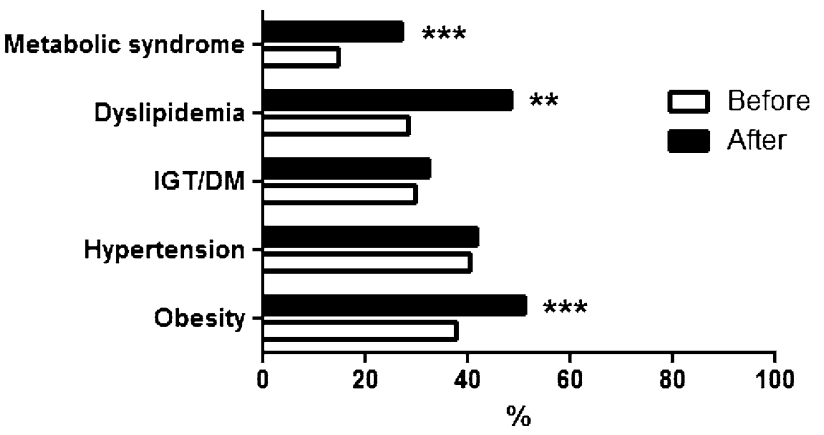

Fig. 1 Changes in the prevalence of metabolic syndrome, dyslipidemia, impaired fasting glucose/diabetes mellitus, arterial hypertension and obesity after COVID-19 outbreak. Consequently to the restrictive measures required for COVID-19 pandemics and total lockdown, prevalence of metabolic syndrome, dyslipidemia and obesity significantly increased in the present cohort of hyperprolactinemic or osteoporotic patients without pre-existing metabolic disorders before COVID-19 outbreak

diseases. None of these patients developed SARS-CoV-2 infection.

At the evaluation after the lockdown period (Fig. 1), 38 patients $(51.3 \%)$ were obese $(p<0.0001), 31(41.9 \%)$ had arterial hypertension $(p=0.995), 24(32.4 \%)$ IGT or DM $(p=0.859)$, and $36(48.6 \%)$ had dyslipidemia $(p=0.003)$. New anti-hypertensive drugs, glucose lowering drugs and lipid lowering drugs were added in 1 (3\%), 5 (20.8\%) and 12 $(33.3 \%)$ patients, respectively. Prevalence of arterial hypertension was significantly higher in group $3(p<0.0001)$ and group $4(p<0.0001)$ as compared to group 1 , and in group $2(p=0.002)$ as compared to group 3. Prevalence of IGT/ DM was significantly higher $(p=0.024)$ in group 2 as compared to group 3. No significant difference was found in the prevalence of dyslipidemia among age groups. Overall MetS was found in $20(27 \%, p<0.0001)$ (Fig. 1). Prevalence of MetS was significantly higher in group $3(p<0.0001)$ and group $4(p=0.043)$ as compared to group 1 , and in group 2 $(p=0.007)$ compared to group 3 .

In our experience, the significant and rapid worsening of cardiometabolic health seen in our patients reflects changes in lifestyle, nutritional habits and social isolation induced by the forced lockdown due to COVID-19 pandemics and affects not only patients with pre-existing metabolic alterations but also those with documented metabolic health before COVID-19. In other words, SARS-CoV-2 outbreak has led to a rapid MetS outbreak, which in turn might furtherly alter immunometabolism and promote chronic systemic inflammation, thus exacerbating hyperinflammation associated with SARS-CoV-2 infection and contributing to COVID-19 increased mortality [6]. Prolonged and sustained stress at one side, and social isolation on the other side have produced detrimental effects on cardiometabolic health. These findings deserve utmost consideration, taking into 
account that the impact of isolation and loneliness on health and mortality are reportedly of the same order of magnitude as other risk factors in COVID-19 patients such as arterial hypertension, obesity, and smoking [7]. Future research will elucidate the role and the burden of COVID-19 pandemics on long-term cardiometabolic sequelae.

Author contributions RSA conceived the study and supervised the manuscript drafting. RSA, RP, AL, RS, and GV performed the literature search, contributed to the interpretation of the data, and prepared the figures. RSA wrote the manuscript. AC provided significant expert contribution in the scientific content revision process. RPiv and AC critically reviewed the manuscript and revised it for important intellectual content. All authors read and approved the final manuscript.

Funding None.

\section{Declarations}

Conflict of interest The authors have no conflicts of interest to declare.

Open Access This article is licensed under a Creative Commons Attribution 4.0 International License, which permits use, sharing, adaptation, distribution and reproduction in any medium or format, as long as you give appropriate credit to the original author(s) and the source, provide a link to the Creative Commons licence, and indicate if changes were made. The images or other third party material in this article are included in the article's Creative Commons licence, unless indicated otherwise in a credit line to the material. If material is not included in the article's Creative Commons licence and your intended use is not permitted by statutory regulation or exceeds the permitted use, you will need to obtain permission directly from the copyright holder. To view a copy of this licence, visit http://creativecommons.org/licenses/by/4.0/.

\section{References}

1. Bracale R, Vaccaro CM (2020) Changes in food choice following restrictive measures due to COVID-19. Nutr Metab Cardiovasc Dis. https://doi.org/10.1016/j.numecd.2020.05.027

2. Estruch R, Ros E, Salas-Salvadó J, Covas MI, Corella D, Arós F, Gómez-Gracia E, Ruiz-Gutiérrez V, Fiol M, Lapetra J, LamuelaRaventos RM, Serra-Majem L, Pintó X, Basora J, Muñoz MA, Sorlí JV, Martínez JA, Martínez-González MA (2013) Primary prevention of cardiovascular disease with a Mediterranean diet. N Engl J Med 368:1279-1290

3. Fang L, Karakiulakis G, Roth M (2020) Are patients with hypertension and diabetes mellitus at increased risk for COVID-19 infection? Lancet Respir Med 8(4):e21

4. King AJ, Burke LM, Halson SL, Hawley JA (2020) The challenge of maintaining metabolic health during a global pandemic. Sports Med 50(7):1233-1241

5. The IDF, Alberti KG, Zimmet P, Shaw J (2005) IDF epidemiology task force consensus group. The metabolic syndromea new worldwide definition. Lancet 366:1059-1062

6. Mauvais-Jarvis F (2020) Aging, male sex, obesity, and metabolic inflammation create the perfect storm for COVID-19. Diabetes. https://doi.org/10.2337/dbi19-0023

7. Cacioppo S, Capitanio JP, Cacioppo JT (2014) Toward a neurology of loneliness. Psychol Bull 140(6):1464-1504

Publisher's Note Springer Nature remains neutral with regard to jurisdictional claims in published maps and institutional affiliations. 